\title{
¿QUÉ IDEAS TIENEN LOS PROFESORES DE FÍSICA DE NIVEL MEDIO RESPECTO AL MODELADO?
}

Stella M aris Islas

M arta A. Pesa ${ }^{2}$

Resumen: En el marco de una investigación referida a explorar qué significado y qué rol atribuyen profesores, estudiantes universitarios e investigadores en Física a los modelos científicos, se presentan en este trabajo los resultados obtenidos con profesores de nivel medio. Se detecta una falta de reflexión sobre el significado y empleo de modelos en Física y serias dificultades para interpretar cómo se vinculan éstos con la realidad y qué rol se les asigna en la construcción del conocimiento científico.

Unitérminos: M odelo Científico; M odelo Pedagógico; Profesores

Abstract: This paper deals with the results obtained through a research work on $\mathrm{H}$ igh School teachers, it is part of an investigation inquiring the significance/meaning and role that teachers, college students and Physics researchers ascribe to scientific models.

A lack of understanding of the meaning and use of models in Physicsand serious drawbacks to interpret how the models are related to reality and the role they are assigned in the construction of scientific knowledge is observed.

Keywords: Scientific model; Teaching M odel; Teachers

\section{Introducción}

Se presentan los resultados obtenidos en una investigación realizada con profesores (graduados universitarios) que dictan clases de Física en escuelas de la ciudad de Tandil (Provincia de Buenos Aires, Argentina).

D esde una postura teórica que coloca a la modelización como una actividad importante en la investigación científica, y considerando que el aprendizaje de la Física demanda la interpretación de situaciones modelizadas, se trató de indagar acerca del modo en que los modelos son introducidos y desarrollados en las escuelas. Para ello, el problema se centró en la formación previa de estos docentes en su carrera universitaria y en la descripción que ellos hacen sobre el rol de los modelos en su actividad educativa. El estudio se realizó con los siguientes propósitos.

- Caracterizar las concepciones de modelo científico sustentadas por Profesores de Física.

- D escribir las experiencias personales de los Profesores en lo referente a model os científi-

cos.

\section{Antecedentes de investigación sobre esta problemática}

En otros reportes de investigación se informa sobre deficiencias en la formación de docentes de Física, debidas a la escasez de instancias de reflexión sobre cuestiones epistemológicas (M ELLAD O , 1997; VAN DRIEL, VERLO O P, 1999; KOULAIDIS, O G BO RN , 1996;

\footnotetext{
${ }_{1}^{1}$ Professora D outora do D epartamento de Formación D ocente, Facultad de Ciencias Exactas, U niversidad $\mathrm{N}$ acional del Centro de la Provincia de Buenos Aires, Tandil, Buenos Aires, Argentina (e mail:sislas@exa.unicen.edu.ar).

2 Professora D outora do D epartamento de Física, Facultad de Ciencias Exactas y Tecnología, U niversidad N acional de Tucumán, Tucumán, Argentina (e-mail: mpesa@herrera.unt.edu.ar).
} 
entre otros). En particular, sobre lo atinente a la modelización científica VAN DRIEL, VERLO O P (1999) encuentran concepciones de modelo científico que muestran inconsistencias, tales como: "sostener una visión positivista de los modelos, por un lado y por otro, estar de acuerdo con la idea de que los model os son construidos en un contexto social".

A ello se suma el señalamiento de la escasez de investigaciones desarrolladas en torno al manejo de modelos científicos en docentes (señaladas en VAN DRIEL, VERLO O P, 1999; M ELLAD O, 1997), y la detección de dificultades en estudiantes de diversas edades para:

- interpretar la relación del modelo con la realidad (ALBAN ESE, VICEN TIN I, 1997;

H ARRISO N, TREAGUST, 1996, 2000; entre otros),

- comprender los vínculos entre el modelo y la teoría

- reconocer el rol esencial de las ideas de los científicos en la elaboración de modelos, y del modelo en la construcción del conocimiento CIENTífICO (S. GILBERT, 1991; GROSSLIGHT et al., 1991; M EYLIN G, 1997).

Estos resultados configuran un panorama más problemático si se los analiza desde una postura epistemológica como la que se adopta en el Encuadre Teórico de este trabajo, donde los model os constituyen las construcciones clave de las explicaciones en ciencias y, más aún, si se toman en cuenta los estudios iniciados por D. H estenes en 1987 y continuados por él mismo, y por autores como I. H ALLO UN (1996), entre otros. Esta línea de investigación encuentra sólidos fundamentos para defender la necesidad de incluir en las actividades áulicas acciones de modelado de situaciones, despliegue de modelos y testeo de su validez, como forma de acercamiento entre el espacio escolar y el de la investigación científica.

C abe preguntarse, entonces, en qué medida los docentes están preparados para realizar una transposición didáctica eficiente de los modelos científicos. En efecto, la construcción de modelos pedagógicos (o modelos de enseñanza, GILBERT et al. 1998 a) supone un proceso complejo en el cual el profesor selecciona críticamente y adapta los niveles de complejidad y abstracción de los modelos de acuerdo al desarrollo cognoscitivo de los estudiantes. La intención del presente trabajo es ofrecer unos primeros resultados al respecto.

\section{Encuadre teórico}

El modelo científico, tanto en el planteo de cuestiones como en las acciones que se despliegan con el afán de resolverlas (BU N GE 1985, pág. 46), es una de las construcciones conceptuales que actúan como orientadoras del quehacer de la Ciencia: para poder formular las preguntas en términos científicos, y para poder decidir con criterios fundamentados cuáles son las fuentes de información que proporcionarán las respuestas, es preciso contar con algún referente teóriCo, puesto que el significado de las conceptuaciones está dado por el marco teórico en que ellas se insertan (CU D M AN I 1992). En el registro que se hace en el campo científico subyace una selección de variables, que lleva a registrar solamente aquellas que - desde la teoría - se conciben como significativas (BUN GE, 1985, pág. 418).

0 tros autores (ej. LAUD AN , 1986; TO U LM IN , 1964; BACH ELARD , 1991) sostie nen opiniones consistentes con lo antedicho en cuanto a la prominente función de los presupuestos teóricos como orientadores de la investigación científica, y acuerdan en reconocer entre las tareas esenciales de la investigación científica a la elaboración, empleo y revisión de los modelos que representan a las entidades y fenómenos en estudio.

Tanto el diseño como el empleo de modelos se realizan ante la necesidad de resolver un problema: a la luz de una cierta teoría, el científico define las variables a incorporar en su modelo y formula hipótesis acerca de las relaciones entre ellas. Frecuentemente entran aquí en juego la elaboración de analogías y la construcción de imágenes mentales (N ERSESSIAN, 1995). 
científico) tal que:

Como resultado, el objeto de estudio queda representado en una forma (M odelo

favorece el estudio del mundo físico con las herramientas científicas disponibles (tanto las conceptuales y formales como las experimentales)

su aplicabilidad a las entidades del mundo físico puede ser evaluada no sólo a través de la experimentación sino también del análisis de su validez teórica

puede encuadrarse de modo sistémico en una teoría convalidada y aceptada por la comunidad científica (en algunos casos, cuando este encuadre no es posible, el modelo puede advertir sobre la necesidad de reformulación de una teoría). 0 bien, cuando no se dispone de una teoría, encuadrarse en un marco teórico formulado como guía de la investigación, el que estará sujeto a revisiones y convalidaciones futuras.

El párrafo anterior caracteriza a la noción de modelo científico que se adopta en este trabajo. $\mathrm{D}$ ada la variedad de connotaciones atribuidas al concepto de modelo desde diferentes perspectivas de investigación (variedad señalada, por ej. en: SAM AJA, 1994; BU N GE, 1985), se considera oportuno explicitar, también, que la relación entre el modelo científico y la porción de la realidad que él representa es conceptualizada como una analogía, esto es, la correspondencia entre los elementos que conforman el modelo y los de la realidad es estructural, y no puntual (BUN GE, 1985; SAM AJA, 1994; TOULM IN 1964).

C omo categorías de análisis de los modelos se adopta la clasificación de GILBERT y colaboradores (1998 a) donde se presentan de forma articulada y relacionada cuatro tipos de modelo: modelo mental, modelo expresado, modelo consensuado y modelo pedagógico. Se denomina modelo expresado a aquél que un científico somete a la consideración de la comunidad con miras a lograr el consenso de la misma; constituye una representación externa del modelo mental que el científico ha construido para la situación representada en el modelo. La aceptación de la comunidad científica posibilita que ese modelo se instale como modelo consensuado. A partir de un modelo consensuado, la comunidad docente (diseñadores de curriculum, autores de libros de texto, docentes a cargo de las clases) realiza transformaciones sobre el mismo, que dan lugar a un modelo pedagógico (o modelo de enseñanza). Sobre este tipo de modelos versan las clases de Física; el propósito de la presentación de model os pedagógicos es el de disparar en los estudiantes la construcción de model os mentales que sean consistentes con el modelo consensuado de referencia.

La Figura 1 muestra una esquematización de lo recién expuesto:

\section{Figura 1: Presentación esquemática del proceso por el cual un modelo cien- tífico es incorporado en la estructura cognitiva de los estudiantes}

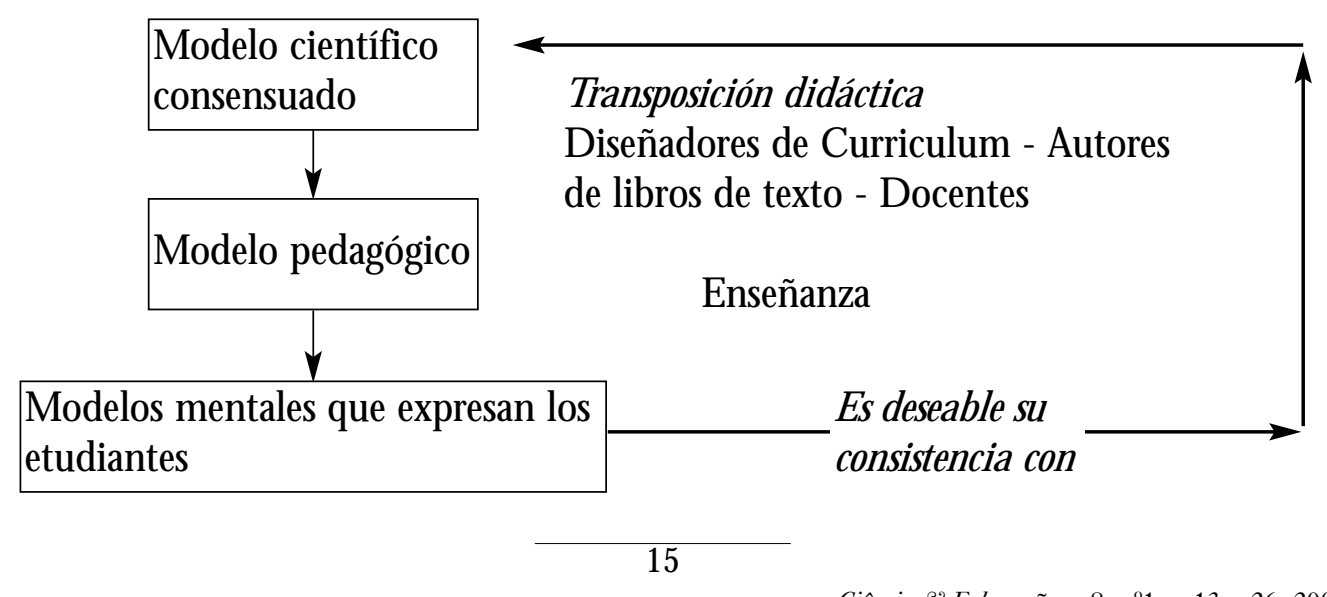




\section{Metodología}

El trabajo es una investigación exploratoria realizada con una metodología cualitativa. D entro de la población conformada por los Profesores que dictan clases de Física en la ciudad de Tandil, se realizó un muestreo de carácter sustantivo (SAM AJA, 1994, p. 270). En este tipo de muestreo, la representatividad de la muestra se basa en el conocimiento de la variabilidad que tienen - en el universo en estudio - los atributos que interesan; desde aquí, se obtiene información acerca de la estructura de ese universo, que permite compararla con la estructura de la muestra a fin de fundamentar la existencia de una analogía entre ella y el universo al cual refiere.

Se conformó la muestra con 6 Profesores, y con ellos se realizaron entrevistas semiestructuradas individuales. Esta estrategia permite registrar, además de las respuestas a preguntas específicas, los comentarios que los entrevistados consideran oportuno agregar.

La información obtenida de la transcripción de las entrevistas ha sido procesada mediante un sistema de categorías de análisis especialmente diseñado para este trabajo. El diseño toma en cuenta las líneas metodológicas de varios autores, principalmente las de D EN ZIN , LIN CO LN (1994) y DEY (1993), y una caracterización sucinta del mismo es la siguiente: sobre la base de reiteradas lecturas de las transcripciones de las entrevistas, se va confeccionando un listado de categorías. Algunas de ellas reúnen las palabras clave de segmentos de texto cuyos significados apuntan en un mismo sentido; otras se han construido a priori de la recolección de datos (ya que se relacionaban con las preguntas de la entrevista); y otras surgen de tópicos agregados por los entrevistados.

La relación entre las variables en estudio y las categorías que se emplearon para compilar la información se muestra en el siguiente esquema (Figura 2):

\section{Figura 2: Análisis de los resultados de las entrevistas ${ }^{3}$}

\section{Variable 1: C oncepto de modelo}

1.1. El modelo y su relación con la realidad

1.2. Validez de los modelos

1.3. Cómo trabajan los científicos con los modelos

\section{Variable 2: Experiencias personales en el manejo de modelos}

2.a) D urante su formación universitaria

\section{2.b) En su trabajo como} docente del nivel preuniversitario
2.1. Iniciación en el manejo de modelos

2.2. D ificultades para el manejo de modelos

2.3. Temáticas en las que mencionam empleo de modelos

2.4. El modelo pedagógico

2.5. Cómo relacionam sus alumnos al modelo con la realidad

\footnotetext{
${ }^{3}$ En las transcripciones de las entrevistas se han realizado al gunas modificaciones sobre los diálogos originales, con el propósito de minimizar la presencia de expresiones idiomáticas propias de la región. D e este modo, se intenta evitar inconvenientes a los lectores que no son argentinos.
} 


\section{¿QUÉ IDEASTIENEM LOS PROFESSORES DE FÍSICA}

C abe iniciar la presentación de este análisis con una consideración general: tanto en el transcurso de algunas entrevistas, como en la tarea de procesamiento, resultó difícil separar las consideraciones acerca del significado de modelo, de los comentarios referidos a la manera en que la modelización es manejada en clase con los alumnos.

Además, consideramos apropiado agregar aquí otro dato respecto de la dinámica de al gunas de las entrevistas, que puede influir en la manera de analizar (y luego, de interpretar) los resultados: hubo dos docentes con los cuales se hizo muy notable la dificultad para hacer fluir el diálogo en torno a la conceptualización de modelo. En ambos casos, se registraron manifestaciones de desconcierto ante el tema. Para poder proseguir con cada entrevista, se apeló al recurso de elevar el nivel de sugerencia de las preguntas y de los ejemplos.

\section{Variable 1: C oncepto de modelo}

\section{C ategoría 1.1. El modelo, y su relación con la realidad}

La idea de modelo como imagen de un hecho real, es caracterizada como "fotografía" en dos de las seis entrevistas, y se lo hace explícitamente, usando esa palabra. En las demás entrevistas, no se encuentran alusiones a este concepto de modelo como imagen.

La noción de modelo como una representación simplificada de los fenómenos reales aparece solamente en dos de las entrevistas. En una de ellas, se percibe un razonamiento inconsistente porque se atribuye simultáneamente al modelo la idea de imagen fotográfica (con toda la complejidad que ella implica), con la de simplificación: “... cuando trabajas en Física, lo que haces es un recorte de la realidad, tomas una situación, y es como si la congelaras, la abstraes y empiezas a eliminar todo lo que no te sirve para la situación que quieres analizar. 0 sea, estás recortando un instante, sacándolo del contexto en el cual estaba... por ejemplo: un automóvil que pasa por ahí, pero le haces una fotografía ahí y tomas la fotografía, no es cierto? Y entonces, empiezas a hacer suposiciones que te sirven. En realidad, estás sacándole todo lo que te molesta (por decirlo de alguna manera), todo lo que te influye, para dejar... el esqueleto, aplicar tus conceptos físicos a ese esqueleto, volverlo después a insertar en la realidad y ver si lo que vos has predicho, se cumple. Eso es modelizar, no? hacer un recorte, sí?".

D e esta entrevista también se infiere que este docente atribuye un carácter estático a los modelos. En efecto, el recorte de la realidad implicaría para él "detener" el sistema, considerar sólo un instante de tiempo, desconociendo la importancia de los modelos explicativos de procesos para predecir el comportamiento espacial y temporal de los sistemas.

En 4 de las 6 entrevistas señalan que el modelo debería contemplar la mayor cantidad posible de variables del hecho en estudio. La concepción de los docentes parece estar próxima a la del modelo como una "copia" de la realidad, por lo cual el modelo debería ser una construcción cuya similitud con el hecho real sea la mayor que puede lograrse.

La escasez de referencias a la simplificación practicada al elaborar un modelo, es coherente con el hecho de que en ningún caso se registren citas acerca de la posibilidad de hacer, sobre un modelo, sucesivos ajustes para acercarlo al hecho que él representa, cuando este acercamiento es demandado por el estudio que se está realizando.

\section{C ategoría 1.2. Validez de los modelos}

La referencia a los límites de validez de cada modelo está en dos entrevistas, aunque no se preguntó en forma directa sobre estos límites. En una de ellas, se registra lo siguiente: "... como siempre decimos nosotros: en un intervalo, se comporta con esas características, no? 0, bajo estas condiciones, esto se va a comportar de tal y cual manera. $Y$ eso es lo que 
nosotros hacemos: al modelo ponerle algunas condiciones para que esa situación real, responda a ese modelo.(...) en algún momento... pareciera ser totalmente disparatado considerar un tema real bajo un modelo con el cual uno no encuentra demasiados puntos de contacto, y sin embargo, cuando resuelve el modelo, y esa ecuación la aplica a la realidad, funciona, no? (...) Lo que pasa es que uno no se puede desprender de la realidad. Cuando uno está tomando (una situación a modelizar para resolver un problema), lo corta, lo vuelca y lo analiza... ahí hay un montón de factores, que fueron eliminados. Y si no, pensemos en cuando le das un problema a resolver a un alumno: se lo recorta tanto que siempre es fantástico; ahora, cuando vas a la realidad, eso le funciona, en forma media, no es cierto? por ejemplo, cuando le hacemos resolver a un alumno un problema de distancia, velocidades, aceleraciones; es una situación totalmente ideal, eso le ocurre solamente en un intervalo pequeñísimo de su recorrido de una cuadra. Y sin embargo, se los damos... porque en términos generales, es más o menos lo que le va a pasar, pero, ¿cuántas cosas has eliminado? Entonces, tu modelo, ¿cuánto está de próximo a la realidad? Y... qué sé yo, está a... (el tono sugiere disgusto) no? hay un abismo entre el modelo y la realidad. Sin embargo, se pueden hacer predicciones, y de hecho, el alumno dice: ' $y$, sí, si voy a tal velocidad, tengo que tener mayor distancia para frenar, etc....'. Y funciona bastante bien, pero te das cuenta de que de la situación que planteaste a la situación real, hay un abismo, porque no tuviste en cuenta cuántas cosas..." .

Puede percibirse de este fragmento la noción de que, pese al aparente apartamiento del modelo con el hecho real que representa, al tomar en cuenta cuáles son los límites de validez de dicha representación, pueden formularse predicciones acerca del comportamiento del fenómeno en estudio y que tales predicciones pueden ser contrastadas con los comportamientos observados. Sin embargo, no se destaca la importancia de la estimación de los valores de esas incertezas como procedimiento fundamental para salvar la brecha entre el modelo y la realidad.

\section{C ategoría 1.3. Cómo trabajan los científicos con los modelos}

En la guía de las entrevistas se presentaba una cuestión que incluía una pregunta directa sobre los propósitos que persigue la elaboración de modelos.

Las aseveraciones de los docentes entrevistados interrelacionan a la elaboración de modelos con el uso que se hace de ellos. A modo de característica común a todas las respuestas puede señalarse que el modelo es interpretado como una herramienta útil para diversos fines. $Y$ en carácter de 'palabras clavé de las opiniones vertidas, pueden marcarse las siguientes:

- responder preguntas (sobre Física Cuántica)

- organizar el conocimiento

- para "entender" el hecho en estudio y comunicarlo

La primera aparece así: "Yo creo que uno se hace un modelo para responderse preguntas. U no se plantea preguntas sobre al go, sobre un fenómeno, y... si no lo modeliza, al menos en ciertas ramas de la Física, creo que no habría manera de responder a esas preguntas". Y luego retorna al tema diciendo: "... hay aspectos de la Física en los que si uno no se plantea un mode lo del cual agarrarse, del cual tener esa foto, esa imagen... creo que uno se haría las preguntas, pero no podría responderlas después. Se perdería en una nebulosa". U na lectura global de esta entrevista permite inferir que con la expresión "aspectos de la Física" que sólo pueden abordarse desde un modelo, esta docente está aludiendo a los temas de Física Cuántica; no se encuentran planteos, en esta entrevista, que consideren el aporte de la modelización en 
otros campos de la Física. Ello implica una falta de toma de conciencia del alto nivel de abstracción de campos de la física clásica tales como estática, dinámica u óptica geométrica y nos llevaría a reflexionar sobre cómo se enseñan estas temáticas donde aparecen modelos con altos niveles de abstracción.

Una interpretación despojada de los significados más importantes del modelo se obtiene en otra entrevista: "... y, sería para ordenar un poco... la ciencia. Pero el problema es que lo tomes como fin último, como algo supremo, que no es posible salirse de un modelo". Y aquí, la conversación regresa a una inquietud muy destacada por esta docente, que es la de no quedar, en sus clases, limitada a cuestiones abstractas y vincular los modelos con situaciones problemáticas referidas a la realidad de los estudiantes.

La recién citada función organizativa de la modelización, se relaciona en otra entrevista con una valorización del carácter abstracto de los modelos: la selección de variables relevantes (y la consecuente eliminación de las no relevantes) centra la atención en los rasgos sustanciales de una situación; aparece aquí el carácter funcional de los modelos por su potencialidad para interpretar otras situaciones que - siendo distintas de la primera en al gunos aspectos - comparten con ella ciertos rasgos sustanciales. Eso se expresa cuando la docente señala: " ... porque una vez que has modelizado, el modelo te sirve para distintas situaciones en las cuales puedas hacer la abstracción, y, salvo pequeñas diferencias, reproduzcas lo mismo, eh... situaciones que pueden aparecer muy distintas en la realidad, cuando se las recorta y se las plantea, responden al mismo modelo, no es cierto? Yo creo que ése es el propósito: para que uno tenga organizados sus conocimientos. $Y$ entonces, pueda aplicarlos en distintas situaciones que parecieran... distintas".

En cuanto al uso de más de un modelo para resolver la misma situación problemática las opiniones recogidas cubren un espectro que va desde la oposición, a la posibilidad de mirar un fenómeno desde dos puntos de vista diferentes, hasta el señalar esta posibilidad como un hecho que deriva de la propia naturaleza del conocimiento científico. Ejemplo de esta última concepción es la siguiente: "Como yo creo que trabaja un científico... debería tener la mente suficientemente abierta como para cambiar de modelo.(...) Y yo juzgaría, incluso... juzgaría al científico que no tiene su mente abierta como para cambiar de modelo; sería al go que no habla bien de ese científico, creo yo, creo que no habla bien de él".

En el párrafo recién transcripto también se está respondiendo afirmativamente a la pregunta sobre la posibilidad de que un científico cambie de modelo. 0 tro docente atribuye mayor flexibilidad para este cambio a los científicos más jóvenes. En ambos casos, la ductilidad en el uso de modelos por parte de los científicos, se presenta relacionada con peculiaridades personales de cada científico. Podría decirse que estos docentes están admitiendo que el modelo construido por un científico no queda "congelado" una vez que lo expone, sino que está sujeto a una permanente revisión desde los resultados de su propio trabajo (aunque no hacen referencias a las influencias de la actividad comunitaria). Sin embargo, merece destacarse que los cambios a que hacen mención se refieren a la aceptación de nuevas teorías y no al uso de distintos modelos en distintos contextos o en la resolución de una misma situación problemática con distintos grados de precisión.

\section{VARIABLE 2: EXPERIENCIAS PERSONALES EN EL USO DE MODELOS}

Se realiza aquí una separación de registros en dos grupos que reúnen: por un lado, las categorías que corresponden a la carrera de formación universitaria de los docentes y, por otro, a las referidas a su actividad laboral. 


\section{2.a) D urante su formación universitaria Categoría 2.1. Iniciación en el manejo de modelos}

Como respuesta a una pregunta directa sobre este punto, en tres entrevistas se manifiesta el reconocimiento de alguna instancia de aprendizaje formal referida a algunos aspectos del modelado que se realiza en la Ciencia.

En un caso, en una breve explicitación de la respuesta, se menciona el recuerdo (no muy preciso) de haber pasado por alguna situación en la que algún Profesor del entrevistado trató el tema de "cómo hacer esos recortes", aludiendo a las decisiones relacionadas con la simplificación que conlleva la elaboración de un modelo.

0 tra de las personas entrevistadas alude más marcadamente a ocasiones en las que los Profesores de la Facultad le proporcionaron elementos para vincular a las entidades físicas con las formulaciones matemáticas involucradas en un modelo, así como para distinguir entre el modelo y la porción de la realidad que él representa.

En una de las entrevistas se muestra al tratamiento de la modelización restringido a los temas de Astronomía durante la carrera universitaria, e inmediatamente se plantea: "... es algo aś como que... en lo último te das cuenta de que son modelos, es como que te decían (los profesores): esto es un modelo. Entonces los ibas estudiando y diciendo: bueno, Fulano dijo esto, y ya está". Esto Ilama la atención hacia un aspecto que, a los fines de este trabajo, se considera de sumo interés, y es que los docentes manifiestan que durante los primeros años de su carrera de formación universitaria en Física, no advertían que estaban usando modelos para abordar el estudio de los contenidos de las asignaturas básicas; declaran haber tomado conciencia de la modelización practicada sobre la realidad, recién cuando estaban avanzados en la carrera. Cuando se adentraron en el estudio de los fenómenos a nivel atómico, o bien (en el otro "extremo" dimensional) cuando discutieron modelos a escala astronómica, vislumbraron la posibilidad de que la modelización alcance a otros campos de la Física.

D os docentes expresan claramente que desconocían que estaban usando modelos cuando estudiaron la física básica: “... yo los usaba, pero no... yo creo que me costaba darme cuenta de que usaba eso como modelos. Sí, imaginar así a veces las cosas... como puntuales, bueno, uno se acostumbra por mecanismo. Pero no es algo... ya es un mecanismo que adquiere, y después, con el tiempo va logrando darse cuenta qué era. A mí me costó darme cuenta de esas cosas. (...) porque uno en la Universidad usaba mucho la ecuación... en el momento que uno estudia, trata de aplicar la fórmula adecuada al problema, y es muy mecánico lo que va haciendo. D espués, cuando uno tiene que estudiar para el examen final, ya sí, uno empieza a razonar más las cosas. (...) Entonces, era un problema, y había que recordar la fórmula, y aplicarla, y uno no pensaba demasiado en el contenido físico."

D e este extracto y de la lectura global de la entrevista puede inferirse que, en opinión de esta docente, la falta de reflexión sobre los modelos empleados atenta contra la compresión física de los problemas que se resuelven, y que la elaboración personal realizada para dotar de contenido físico a los "mecanismos" utilizados en la resolución de problemas incluye la toma de conciencia de los model os que están tácitamente involucrados en tales procesos de resolución. Puede verse aquí a la comprensión del modelo como una conquista que aporta elementos para evitar que el manejo de formalismos quede vacío de contenido fáctico.

Una aseveración de otro tono, referida a lo anterior, se da en otra entrevista. $M$ ientras se está tratando el tema de la relación entre un modelo y la porción de la realidad a la cual él representa, la docente enfatiza la necesidad de hacer una "bajada a la realidad" 
mediante la discusión de problemáticas referidas al entorno cotidiano de los estudiantes, y luego indica: "Yo no sé... a mí me parece mucho más importante todo esto, y no que ellos sepan de memoria modelos, o... que sepan fórmulas". En éste, y en otros tramos de esta entrevista, aparece la confusión entre el significado de modelo en cuanto a su potencialidad para comprender y explicar las situaciones físicas y la simbología matemática y los formalismos.

\section{Categoría 2.2. D ificultades para el manejo de modelos}

Las respuestas muestran las dificultades relacionándolas con el grado de abstracción de aquellas temáticas en las cuales fue más explícito el manejo de modelos. La mayoría de los docentes entrevistados afirmaron que estas temáticas son las de Física Atómica y N uclear. Y pueden reconocerse señalamientos en los siguientes sentidos:

- vincular la imagen asociada al modelo, y los formalismos matemáticos correspondientes al mismo

- encontrar "si tuaciones de la realidad" a las cuales pueda aplicarse el modelo (cuántico) en estudio

- poder cambiar la imagen creada desde un modelo sencillo, para utilizar otro de mayor complejidad

\section{Categoría 2.3. Temáticas en las que mencionan empleo de modelos}

Los temas citados por mayor número de docentes son los de Física Atómica y M ecánica Cuántica. Predomina, entre los ejemplos, la mención del modelo atómico, y la referencia a las asignaturas más avanzadas de la carrera.

Sólo hay dos entrevistas en las que se muestra a la modelización cubriendo un espectro temático más amplio, que es el de las asignaturas que en esta Facultad se han dictado con los nombres de "Física I y II", y que abarcan los tratamientos de la M ecánica newtoniana y del Electromagnetismo clásico, respectivamente. En estos dos casos puede advertirse una concepción que sitúa a la construcción de modelos como una heurística aplicable al estudio de diferentes aspectos de la realidad.

En las restantes entrevistas, la modelización no parece estar conceptualizada como una actividad inherente a la Física en su totalidad, sino más bien, como una elaboración destinada a comprender ciertos fenómenos (los que ocurren a nivel microscópico), y a volver accesible el estudio de otros (aquellos cuya complejidad demanda recortar variables)

\section{2.b) En su trabajo como docente del nivel preuniversitario Categoría 2.4. El modelo pedagógico}

Los resultados de las entrevistas muestran claramente una falta de toma de conciencia respecto al uso de los modelos en la enseñanza y el aprendizaje de la física. Los docentes utilizan modelos, pero no son conscientes de que los usan.

En efecto, el empleo de modelos no se señala en todos los casos como una actividad frecuente en el aula: una de las entrevistadas afirma que no usa modelos en sus clases, y una idea similar puede inferirse del estudio de otras dos entrevistas. En estos tres casos, se deben interpretar los resultados teniendo en cuenta que hubo dificultades para situar a los entrevistados en el tema del modelado.

En las otras tres entrevistas, es reconocido un uso constante de modelos en las actividades de aprendizaje de la Física, dada la necesidad de recortar las situaciones reales para poder abordar su estudio. Pero este uso no es, para algunos docentes, realizado de 
manera consciente, ni es explicado a los alumnos; véase el siguiente ejemplo: "... tal vez, lo que no me he planteado es si... no lo he pensado: esto lo uso como modelo, y tal vez no la palabra modelo. Pero los uso, sí, uno siempre usa modelos, sí".

Este carácter tácito del empleo de modelos es resaltado en otra ocasión, como una acción no conveniente: "en realidad no les estás diciendo (el profesor a los alumnos) que le estás haciendo un model $0 .$. . se les dice que estamos tratando de resolver esta situación, sin decirle que se la está modelizando".

Estos docentes están describiendo su proceder frente a sus alumnos como similar al de quienes fueron sus profesores en las Físicas básicas de la U niversidad: Ios modelos se emplean permanentemente, pero los alumnos no perciben la presencia de la modelización.

Además del estilo implícito para los estudiantes (iy también para los docentes!) del empleo de los modelos en sus clases, los docentes también tienen dificultados para explicitar la construcción de los modelos pedagógicos en el proceso de transposición didáctica. Al solicitarles ejemplos de los modelos que emplean en el aula nombran solamente: "Ios temas de Física atómica y nuclear" (aunque no se puntualiza cuáles modelos se toman en ese campo y cómo se adaptan los niveles de complejidad y abstracción de estos modelos a las características de la estructura cognoscitiva de los alumnos), y "el modelo de cuerpo puntual" para los estudios cinemáticos de los movimientos.

Se halla una única expresión en la cual se trata el tema de la relación del modelo científico con el pedagógico, exponiendo la necesidad de combinar el rigor científico con la accesibilidad a los estudiantes: "Entonces yo, como docente, debería estar capacitada para ele gir el model o que se adapte... queél (el estudiante) lo pueda asumir mejor, y además, por supuesto, que esté lo más próximo, lo más cercano posible a lo que es en realidad el mundo de la Física; pero que seguramente no va a coincidir con el del físico".

En las restantes entrevistas, no aparecen consideraciones de este estilo; sin embargo, una lectura global de todas ellas permitiría inferir que los demás docentes estarían de acuerdo con lo antedicho.

\section{C ategoría 2.5. C ómo relacionan sus alumnos al modelo con la realidad}

Se percibe insatiffacción de estos docentes respecto del modo en que sus alumnos se manejan en el tema de la modelización. Esta insatisfacción, empero, debe ponderarse desde la óptica de los resultados anteriormente descriptos, que muestran a la modelización como un tópico que no ha sido reflexionado con detenimiento por parte de al gunos de estos docentes. Las declaraciones recogidas señalan diferentes dificultades que detectan en sus alumnos, y se reseñan a continuación.

Cuando una de las entrevistadas está manifestando que valora las estrategias empleadas por quienes fueron sus profesores en la Universidad, que lograron hacerle notar los vínculos entre las formulaciones matemáticas y las entidades físicas a las que refieren, se pregunta a sí misma si ella tiene el mismo éxito con sus alumnos y unos párrafos más adelante, concluye que esto es muy difícil de conseguir.

0 tra dificultad que manifiestan estos docentes es que sus alumnos identifican al modelo con la realidad. A la misma docente cuyas preocupaciones se comentaron en el párrafo anterior, se le preguntó sobre este punto mientras está hablando del empleo de modelos del núcleo atómico, y el diálogo se desenvuelve del siguiente modo:

S: ¿y tú crees que ellos entienden que eso es un modelo?

D : no (categóricamente) yo creo que se creen que es así. Creo que ni siquiera creen lo que uno... uno intenta decirles, les dice que no es así, pero, no, pobres, no... 


\section{¿QUÉ IDEASTIENEM LOS PROFESSO RES DE FÍSICA}

S: ¿no lo entienden porque son muy niños?

$\mathrm{D}$ : claro, es primer año Polimodal! ${ }^{4}-\mathrm{N} 0$ creo que eté mal, no!. N o creo que esté mal. Yo me pongo en el lugar de ellos y... intento recordar, tantas cosas de Física Cuántica, que uno intentaba entender, y... uno se agarró del modelo, eh? y le hizo bien agarrarse del modelo. Yo creo que uno después lo reconstruye; pero si uno en el momento no se agarra del model o... no hay manera de entender cómo viene... cómo funciona... Q uizás, lo que está mal es que se queden nada másque con eso, y que después, en algún otro momento de su vida, no profundice y no diga: ah! pero esto no es aś; ; ahí está el asunto. Por eso te digo... viéndolos en Física Cuántica, a mí, en su momento, me servía agarrarme del modelo. Pero... si después uno no se lo re plantea... si los alumnos se quedan nada más que con eso, creo que se está haciendo un mal. Ese modelo es el que más... ya te digo, el tema de Física Nuclear".

En este caso, la identificación del modelo con la realidad se advierte como problemática para los tópicos de Física N uclear, que son los únicos para los cuales se consideran modelos en esta entrevista. Por otro lado, esta docente ha planteado al carácter representacional del modelo como una imagen que, en su experiencia personal, ha sido válida para llenar de contenido físico a las formulaciones matemáticas de la Física Cuántica. Pero no queda clara cuál sería la función del modelo para sus alumnos, que no estudian tales formulaciones. Sin embargo, reitera la manifestación de una preocupación por lograr que sus alumnos entiendan que el modelo no es la realidad, pero que es una herramienta útil para estudiarla.

En el Encuadre Teórico de este trabajo se mencionaron algunos de los estudios (ALBAN ESE, VICEN TIN I, 1997; H ARRISO N , TREAGUST, 1996, 2000) que han registrado esta confusión ontológica al investigar sobre modelos representativos de entidades no accesibles a la percepción sensorial. Al identificar, por ejemplo, el átomo con un modelo del mismo, los estudiantes creen que sólo existe un modelo del átomo que es el "correcto"; algo similar ocurre cuando se trata de explicar otras entidades tales como los genes, los enlaces interatómicos, las "nubes" electrónicas, etc. (H ARRISO N, TREAGU ST, 2000).

En otros diálogos se verbalizan preocupaciones similares a la anterior, pero dirigidas a otras temáticas, ya que aluden al recurso al modelo para estudiar situaciones que los estudiantes conocen en su entorno cotidiano (ejemplo: el modelo de cuerpo puntual para describir movimientos de objetos observables).

\section{Interpretación de los resultados}

La modelización que se realiza en la investigación en Física aparece como un asunto poco reflexionado por los docentes aunque (en un modo un tanto paradojal) reconocen su valor como constructo que permite abordar el estudio de la naturaleza, y se muestran preocupados por el modo en que sus alumnos manejan estas cuestiones. El carácter comunitario de la construcción del conocimiento científico no se presenta, tampoco, como una cuestión meditada.

Estos hechos muestran una distancia entre la postura docente y la de un investigador ya que este último, en su trabajo habitual diseña, despliega, evalúa y corrige modelos científicos a la luz de las teorías y los resultados de las convalidaciones experimentales, teniendo en cuenta el consenso comunitario.

La noción de modelo como una construcción teórica en cuya elaboración es central la simplificación del sistema real a representar, aparece en algunos de los discursos de

\footnotetext{
${ }^{4}$ edad promedio de los estudiantes: 14 años
} 
los profesores entrevistados. No ocurre lo mismo con el carácter analógico de la relación entre modelo y realidad: el modelo se presenta, en varios casos, como una representación que intenta "copiar" a la realidad; en este último aspecto las concepciones de modelo que subyacen al discurso docente no son consistentes con las que se hallan consensuadas en la literatura de investigación educativa.

Los docentes entrevistados en este estudio, desde la concepción de modelo como copia de la realidad, tienden a establecer una correspondencia "uno a uno" entre cada modelo y el objeto (o el fenómeno) de la realidad modelizada.

D esde esa postura, les resulta difícil aceptar que el modelo que se elabora para repre sentar a un cierto sistema real puede variar- como ya dijimos anteriormente según el encuadre, los propósitos y las condiciones particulares del estudio de tal sistema (de aquí surgen los "múltiples modelos" a los que aluden GROSSLIGH T et al. [1991], H ARRISO N, TREAGUST [2000]).

Además, sólo al gunos de estos docentes evidencian haber reflexionado sobre la posibilidad de que un mismo modelo sea apto para el estudio de más de un sistema real; aunque todos reconocen que el diseño del modelo toma en cuenta solamente las variables relevantes de un sistema dado, el modelo se concibe como algo muy concreto y válido para una sola situación.

Esto nos inclina a pensar que no se advierte que un modelo de alto nivel de abstracción puede ser utilizado para interpretar muchos fenómenos y que, asimismo, un cierto sistema real puede modelarse de diferentes maneras.

"El tener más de un modelo para un cierto sistema, y más de un sistema representado por un cierto modelo, sugiere la existencia de un continuo de posibles modelos que va desde los muy concretos hasta los muy abstractos, que pueden explicar los fenómenos. Un modelo muy específico puede explicar o describir sólo un fenómeno particular, y un modelo muy general puede explicar muchos fenómenos". (SN YD ER, 2000).

Las concepciones de modelo de estos docentes parecen estar muy alejadas de la noción consignada por Snyder.

Las experiencias personales de estos docentes en relación con la modelización científica parecen haber tenido un comienzo similar al de sus actuales alumnos: durante el estudio de las Físicas básicas el empleo de modelos no es advertido; el modelado se evoca en relación con ciertos campos de la Física y no, como una característica del conocimiento científico. Estas dificultades han sido señaladas también en otros estudios que detectan la falta de formación de los docentes en cuestiones epistemológicos (ej. VAN DRIEL, VERLO O P, 1999) así como la escasa vinculación de los docentes en ejercicio con los investigadores científicos, tanto con los que se dedican a la Física (VIAN N A, C ARVALH O , 2000) como con quienes realizan investigación sobre educación científica (SH KEDI, 1998).

Es posible, entonces, que la resignificación de los modelos científicos en las aulas se esté llevando a cabo, en muchos casos, sin una previa consideración de las transformaciones que se operan para construir un modelo pedagógico basado en un modelo científico consensuado.

\section{CONCLUSIONES}

Las concepciones de modelo científico que manifiestan los docentes entrevistados difieren de los significados otorgados al modelo científico en la comunidad científica y en la literatura sobre educación científica y sobre epistemología de la Física. 


\section{¿QUÉ IDEASTIENEM LOS PROFESSORES DE FÍSICA}

La mayoría de ellos no destaca la noción de modelo como una representación simplificada de la realidad y que guarda con la misma una relación de analogía, sino que concibe al modelo como una copia de la realidad. En consistencia con ello, algunos docentes entienden que existiría un único modelo para cada sistema real (el "mejor" modelo posible, aquél que copia al sistema real con mayor grado de detalle), sin tener en cuenta que la representación del sistema real focaliza la atención en aquellos aspectos estructurales que son de interés para el problema concreto a resolver, y que son los contemplados en la construcción del modelo.

Las referencias que -espontáneamente se dan acerca de los límites de validez de los modelos son poco frecuentes, y no hacen hincapié en la medición ni en el cálculo de errores.

El trabajo muestra a la modelización en Física como un tema que no ha sido motivo de reflexión para la mayoría de estos docentes. Esta conclusión merece ser atendida, si se toma en cuenta lo señalado en el Encuadre Teórico respecto de la importancia reconocida a la modelización en la actividad científica, y el interés de la investigación educativa por promover una mejora de los aprendizajes estudiantiles en este aspecto.

Los profesores entrevistados frecuentemente circunscriben el modelado científico a algunos campos de estudio de la Física mostrando, además, confusiones entre el significado de los modelos representacionales y los modelos matemáticos.

Las carreras de formación docente, así como las actividades de capacitación de docentes en servicio, podrían mejorar la preparación de los profesores implementando espacios de discusión sobre cuestiones epistemológicas, y de interacción con científicos en actividad.

\section{Referências Bibliográficas}

BACH ELARD , G. La formación de espíritu científico. Traducción José Babini. 17 ed. Siglo XXI Editores. M éxico: 1991. 302 p.

BU N GE, M. La investigación científica. Su etrategia y su filosofía. Traducción M anuel Sacristán. 2 ed. Barcelona: Ed. Ariel. 1985. 933 p.

CROSS, R. I deology and science teaching: teachers' discourse. International Journal of Science Education, v. 19, o 5, p. 607 - 616, 1997.

CUD M AN I, L. C. Importancia del contexto en la conceptualización científica. Implicancias para el aprendizaje. CET. Revista de la Facultad de Ciencias Exactasy Tecnología. Tucumán. oㅡ 2, p. $13-17,1992$.

CUDM AN I, L. C. de; SALIN AS, J. ; PESA, M . M odelo y realidad M aterial de trabajo para el Taller desarrollado durante la VII Reunión Nacional de Educación en Física. M endoza, 1991.

DEN ZIN , N .; LIN C O LN , Y. (Ed.) H andbook of Q ualitative Research. Thousand O aks,

California: Ed. Sage Publications. 1994, 643 p.

DEY, I. Q ualitative D ata Analysis London: Ed. Routledge, 1993, 304 p.

GILBERT, S. W. M odel building and a definition of Science. Journal of Research in Science Teaching. v. 28, no 1, p. $73-79,1991$.

GILBERT, J. K.; BO ULTER, C.; RUTH ERFO RD, M . M odels in explanations, Part 1: H orses for courses? International Journal of Science Education. v. 20, no 1, p. 83 - 97, 1998.

GILBERT, J. K.; BO ULTER, C.; RUTH ERFO RD , M . M odels in explanations, Part 2:

Whose voice? Whose ears? International Journal of Science education. v. 20, № 2, p. 187 - 203, 1998. 
GROSSLIGHT, L.; U N GER, C.; JAY, E. Understanding M odels and their U se in Science: Conceptions of $\mathrm{M}$ iddle and $\mathrm{H}$ igh School Students and Experts. Journal of Research in Science Teaching, v. 28, no 9, p. 799 - 822, 1991.

H ALLOUN , I. Schematic M odeling for M eaninful Learning of Physics. Journal of Research in Science Teaching. v. 33, o 9, p. 1019 - 1041, 1996.

H ARRISON , A.; TREAGUST, D. Secondary students mental models of atoms and molecules: implications for teaching science. Science Education. London. v. 80, no 5, p. 509 - 534, 1996. H ARRISON , A. ; TREAGUST, D. A typology of school science models. International Journal of Science Education. v. 22, no 9, p. 1011 - 1026, 2000.

HEST EN ES, D. M odeling games in the N ewtonian World. American Journal of Physics v. 60, ำ 8, p. $732-748,1992$.

KOULAIDIS, V.; O GBORN , J. Science teachers' philosophycal assumptions: how well do we understand them? International Journal of Science Education. v. 17, no 3, p. 273 - 283, 1995. M ELLAD 0, V. Preservice Teachers' Classroom Practice and their Conceptions of the $N$ ature of Science. Science \& Education. Dordretch. v. 6, p. 331 - 354, 1997.

M EYLIN G, H. H ow to Change Students' Conceptions of the Epistemology of Science. Science \& Education. Dordretch. v. 6, p. 397 - 416, 1997.

M OREIRA, M. A. M odelos M entais. Investigações em Ensino de Ciências. Porto Alegre. v. 1, no 3, p. 193 - 232, 1998. Disponible en: http://www.if.ufrgs.br/public/ensino/revista.htm N ERSESSIAN, N. Should Physicists Preach What They Practice? Science \& Education. v. 4, no 3, p. 203 - 226, 1995.

SAM AJA, J. Epistemología y M etodología. 2 ed. Buenos Aires: Ed. Eudeba. 1994, 413 p. SN YDER, J. An investigation of the knowledge structures of experts, intermediates and novices in physics. International Journal of Science Education. v. 22, no 9, p. 979 - 992, 2000.

SH KEDI, A. Teachers' attitudes towards research: a challenge for qualitative researchers. International Journal of Qualitative Studies in Education. v. 11, № 4, p. 559 - 577, 1998. TO U LM IN , S. La comprensión humana. V.1 (El uso colectivo y la evolución de conceptos). Traducción N éstor M íguez. Alianza Editorial. M adrid: 1977, 523 p.

TO U LM IN , S. La Filosofía de la Ciencia Los libros del M irasol. Tradução de José Babini. Buenos Aires: Ed. Compañía General Fabril Editora. 1964. 161 p.

VAN DRIEL, I. ; VERLO O P, N. Teachers' knowledge of models and modelling in science. International Journal of Science Education. v. 21, no 11, p. 1141 - 1153, 1999

VIAN N A, D . M .; CARVALH O , A. M . P. Formação permanente: a necessidade da interação entre a ciência dos cientistas e a ciência da sala de aula. Ciência \& Educação. v. 6, no 1, p. 31 42,2000

\section{Artigo recebido em 5 de março de 2001 e selecionado para publicação em 8 de janeiro de 2002.}

